\title{
Pareto optimum by independent trials
}

\section{D.J. Gates and J.A. Rickard}

\begin{abstract}
We consider a method by which players in a continuous $N$-person game can arrive at a Pareto optimal solution by a trial process. The process has a number of novel features. Firstly, it is assumed that the players do not know the payoff functions. Secondly, the players are assumed to act quite independently. In spite of this lack of information and lack of cooperation, the players eventually arrive at what is usually regarded as a cooperative solution. The process is a model of the accounting procedures used by firms, and the results predict approach to an equilibrium state of a market model. Proofs are given only in outline here.
\end{abstract}

\section{The trial process}

We consider an $N$-person continuous game in which the strategy of player $i \quad(i=1, \ldots, N)$ is to choose a value of the real number $\sigma_{i}$. The combination $\left(\sigma_{1}, \sigma_{2}, \ldots, \sigma_{N}\right)$ of strategies of the $N$ players is denoted by $\sigma$. The payoff to player $i$ for such a combination is denoted by $J_{i}(\sigma)$. It is supposed that the payoff functions are not known to the players. However, each player naturally knows what payoff he receives after a play of the game.

The players are to make 2 different moves $\sigma(t-1)$ and $\sigma(t)$ chosen at random at times $t-l$ and $t$. Each player ignores the moves of his competitors. He tries to make an estimate of his own payof $f$ as a function

Received 25 November 1974. 
of his own strategy alone. That is he constructs an estimated payoff function $\tilde{J}_{i}\left(\sigma_{i} \mid t\right)$ of his strategy $\sigma_{i}$ at time $t$.

He does this as follows. He takes his payoffs $J_{i}[\sigma(t-1)]$ and $J_{i}[\sigma(t)]$ resulting from the random moves, and calculates the marginal payoffs, defined by

$$
m_{i}(t-1)=J_{i}[\sigma(t-1)] / \sigma_{i}(t-1)
$$

$$
m_{i}(t)=J_{i}[\sigma(t)] / \sigma_{i}(t) .
$$

Then he constructs an estimated marginal payoff finction $\tilde{m}_{i}\left(\sigma_{i} \mid t\right)$ by drawing a straight line through the two points $\left\{\sigma_{i}(t-1), m_{i}(t-1)\right\}$, $\left\{\sigma_{i}(t), m_{i}(t)\right\}$. This yields

$$
\tilde{m}_{i}\left(\sigma_{i} \mid t\right)=m_{i}(t)+\frac{\Delta m_{i}(t)}{\Delta \sigma_{i}(t)}\left[\sigma_{i}-\sigma_{i}(t)\right],
$$

where

$$
\begin{aligned}
& \Delta \sigma_{i}(t)=\sigma_{i}(t)-\sigma_{i}(t-1), \\
& \Delta m_{i}(t)=m_{i}(t)-m_{i}(t-1) .
\end{aligned}
$$

Next, he takes his estimated (total) payoff function to be

$$
\begin{aligned}
\tilde{J}_{i}\left(\sigma_{i} \mid t\right) & =\sigma_{i} \tilde{m}_{i}\left(\sigma_{i} \mid t\right) \\
& =m_{i}(t) \sigma_{i}+\frac{\Delta m_{i}(t)}{\Delta \sigma_{i}(t)}\left[\sigma_{i}{ }^{2}-\sigma_{i} \sigma_{i}(t)\right] .
\end{aligned}
$$

The player has therefore formed a quadratic estimate of the dependence of his payoff on his own strategy. Naturally, it is usually a crude estimate of the true payoff. However, it appears to be the best that a player can do, given only his moves $\sigma_{i}(t-1)$ and $\sigma_{i}(t)$ and his resulting payoffs $J_{i}[\sigma(t-1)]$ and $J_{i}[\sigma(t)]$.

Using the quadratic estimate (1.4), the player chooses his new strategy $\sigma_{i}(t+1)$ at time $t+1$ to be simply the turning point of $\tilde{J}_{i}\left(\sigma_{i} \mid t\right)$. That is, he chooses 
(1.5) $\quad \sigma_{i}(t+1)=\frac{3}{2}\left[\sigma_{i}(t)-\frac{\Delta \sigma_{i}(t)}{\Delta m_{i}(t)} m_{i}(t)\right]$ if $\Delta m_{i}(t) \neq 0$.

Consider the case $\Delta m_{i}(t)=0$. This means that the marginal payof of player $i$ is the same for times $t-1$ and $t$. In this situation, there is no reason for him to change his strategy. We therefore suppose that

$$
\sigma_{i}(t+1)=\sigma_{i}(t) \text { if } \Delta m_{i}(t)=0
$$

For his next move the player uses the moves $\sigma_{i}(t)$ and $\sigma_{i}(t+1)$, and the resulting payoffs $J_{i}[\sigma(t)]$ and $J_{i}[\sigma(t+1)]$. This gives a formula corresponding to $(1.5)$ for $\sigma_{i}(t+2)$, and so on. This completely specifies the trial process by which we suppose that the game is played.

A few points should be noted. Each player uses only his own data (strategies and payoffs) so that the players are acting independently. The choice (1.5) is naturally motivated by seeking the maximum of the estimated payoff function $\tilde{J}_{i}\left(\sigma_{i} \mid t\right)$. That is, each player would like to maximize his current estimate of his payoff. Without suitable conditions, one cannot guarantee that (1.5) gives a maximum rather than a minimum (see [1]). For simplicity here, we just take (1.5) and (1.6) as the definition of the trial process.

The process we have described is a simplified model of an accounting procedure which might be employed by firms competing in a market. Suppose firm $i$ produces an amount $\sigma_{i}$ of goods in one business period, and sells them all at a price $m_{i}(\sigma)$ determined by the buyers. Its profit, ignoring costs, is

$$
J_{i}(\sigma)=\sigma_{i} m_{i}(\sigma)
$$

Using its production from two initial business periods and its resulting profits, it could determine its new production for the next business period by using (1.5), and continue in this manner. A more realistic version of this process is considered in reference [1]. The process is in fact in the same spirit as the accounting practices actually employed by firms (see We inberg [3]). 


\section{The equilibrium state}

In this section we look for possible equilibrium solutions $\bar{\sigma}$ of the difference equations (1.5). These are defined by the condition that, if $\sigma(t)$ is a solution of ( 1.5$)$, then

$$
\sigma(t) \rightarrow \bar{\sigma} \text { as } t \rightarrow \infty \text {. }
$$

We give an outline of the method for determining such equilibrium solutions (see [1] for details).

Near the equilibrium, $\sigma(t)$ changes slowly, so that

$$
\begin{aligned}
& \dot{\sigma}(t) \text { approximates } \Delta \sigma(t) \text { or } \Delta \sigma(t+1), \\
& \dot{m}_{i}(t) \text { approximates } \Delta m_{i}(t) \text {. }
\end{aligned}
$$

Consequently (1.5) becomes

$$
2 \dot{\sigma}_{i} \dot{m}_{i}+\sigma_{i} \dot{m}_{i}+\dot{\sigma}_{i} m_{i} \approx 0
$$

Since $m_{i}(\sigma)$ depends on all the $\sigma_{i}^{\prime}$ 's, we have

$$
\dot{m}_{i}=\sum_{j=1}^{N} \frac{\partial m_{i}}{\partial \sigma_{j}} \dot{\sigma}_{j} .
$$

Similarly,

$$
\begin{aligned}
\sigma_{i} \dot{m}_{i}+\dot{\sigma}_{i} m_{i} & =\frac{d}{d t}\left(\sigma_{i} m_{i}\right) \\
& =\frac{d J}{d t} \\
& =\sum_{j} \frac{\partial J}{\partial \sigma_{j}} \dot{\sigma}_{j} .
\end{aligned}
$$

Substituting (2.3) and (2.4) in (2.2) yields

$$
2 \sum_{j} \frac{\partial m_{i}}{\partial \sigma_{j}} \dot{\sigma}_{i} \dot{\sigma}_{j}+\sum_{j} \frac{\partial J}{\partial \sigma_{j}} \dot{\sigma}_{j} \simeq 0 .
$$

Since $\dot{\sigma}$ is small, we neglect quadratic terms and obtain

$$
\sum_{j} \frac{\partial J_{i}}{\partial \sigma_{j}} \dot{\sigma}_{j} \simeq 0
$$

This possesses non-trivial solutions for $\dot{\sigma}$ only if 


$$
\operatorname{det} \frac{\partial J_{j}}{\partial \sigma_{j}}=0
$$

that is, the Jacobian of the payoff functions must vanish. The condition defines a set of points in the $N$-dimensional space of $\sigma$. In general it will be an ( $N-1$ - -dimensional surface (or a number of disconnected surfaces). We deduce that the equilibrium solution $\bar{\sigma}$ must be on this surface.

For suitable payoff functions, (2.7) is equivalent to the statement that $\bar{\sigma}$ be a Pareto optimal strategy (see Shubik [2]).

The result can be explicitly demonstrated for the special case of the game

$$
J_{i}(\sigma)=\sigma_{i}\left[a-b \sigma_{T}\right] \text {, }
$$

where

$$
\sigma_{T}=\sum_{i=1}^{N} \sigma_{i}
$$

Economically, this corresponds to a market in which there is a common price $a-b \sigma_{T}$ for all firms. The price decreases linearly as total production increases. Now $(1.5)$ becomes

(2.9) $\quad \sigma_{i}(t+1)=\frac{1}{2}\left[\sigma_{i}(t)+\frac{\left\{a-b \sigma_{T}(t)\right\}}{b \Delta \sigma_{T}(t)} \Delta \sigma_{i}(t)\right]$ if $\Delta \sigma_{T}(t) \neq 0$.

Adding these equations gives

$$
\sigma_{T}(t+1)=\frac{a}{2 b} \text { if } \Delta \sigma_{T}(t) \neq 0
$$

Now consider the Jacobian of (2.8). This is just the determinant of the matrix with elements

$$
-b \sigma_{i}+\left(a-b \sigma_{T}\right) \delta_{i j}
$$

which has the value

$$
\left(a-b \sigma_{T}\right)^{N-1}\left(a-2 b \sigma_{T}\right)
$$

Referring to (2.10), we see that $\sigma_{T}(t+1)$ lies on the surface where 
the Jacobian is zero. Consequently, the players arrive at a Pareto optimal solution after the first application of the trial.

After the next trial there are two possibilities.

(i) If $\sigma_{T}(t)$ happens to lie on the Pareto optimal surface then $\Delta \sigma_{T}(t+1)=0$. It follows from (1.6) that

$$
\sigma_{i}(t+2)=\sigma_{i}(t+1)
$$

Consequently, after the first move to a (possibly new) point on the Pareto optimal surface, the players remain at this latter point for all time.

(ii) In the more usual situation where $\sigma_{T}(t)$ does not lie on the Pareto optimal surface, we obtain at the second application of the trial process (as in (2.10))

$$
\sigma_{T}(t+2)=\frac{a}{2 b} \text { since } \Delta \sigma_{T}(t+1) \neq 0 .
$$

It follows from $(2.10)$ and $(2.14)$ that $\Delta \sigma_{T}(t+2)=0$. Hence from (1.6) we have

$$
\sigma_{i}(t+3)=\sigma_{i}(t+2)
$$

In this case, the first trial results in a point on the Pareto optimal surface; the second trial results in another point on the Pareto optimal surface, and henceforth o remains at this point.

Therefore, we have proved, in the special case (2.8), that the trial process defined by (1.5) and (1.6) leads to an equilibrium strategy which is Pareto optimal. Also, the equilibrium is reached surprisingly rapidy in this case.

\section{References}

[1] D.J. Gates, J.A. Rickard and D.J. Wilson, "Properties of a priceadjustment process", (in preparation).

[2] Martin Shubik, Strategy and market stmucture; competition, oligopoly, and the theory of games (John Wiley \& Sons, New York, London, 1959). 
[3] Robert S. Weinberg, "The uses and limitations of mathematical models for market planning", Mathematical models and methods in marketing, 3-34 (Irwin Series in Quantitative Analysis for Business. Irwin, Homewood, Illinois, 1961).

Department of Mathematics,

University of Melbourne,

Parkville,

victoria. 